\title{
SIMPLIFIED SOLVING PROCEDURE FOR THE ONE-DIMENSIONAL SCHRÖDINGER EQUATION WITH ASYMMETRICAL BOUNDARY CONDITIONS
}

\author{
H. Puszkiarski* \\ Surface Physics Division, Faculty of Physics, Adam Mickiewicz University \\ Umultowska 85, 61-614 Poznań, Poland
}

(Received July 1. 1999)

\begin{abstract}
We propose a new simplified procedure for finding the analytical solutions of the stationary one-dimensional Schrödinger equation, with asymmetric boundary conditions imposed on the equation. The essence of the method consists in expressing the general solution explicitly in terms of the boundary parameters in a form which, by itself, satisfies one of the boundary conditions involved; then, the other boundary condition gives straightforwardly the characteristic equation. This method may tum out to be beneficial with regard to the recently growing interest in one-dimensional quantum systems.

PACS numbers: 03.65. Ge, 02.60.Lj
\end{abstract}

\section{General solution for constant potential}

Let us consider the one-dimensional Schrödinger equation (with constant potential) defined throughout the closed interval of the variable $z \in[-d,+d]$. The equation and the boundary conditions have the following form:

$$
\begin{array}{ll}
\left(-\frac{\mathrm{d}^{2}}{\mathrm{~d} z^{2}}+V\right) u(z)=E u(z), \\
\widehat{S}_{-} u(z)=0 & \text { for } z=-d, \\
\widehat{S}_{+} u(z)=0 & \text { for } z=+d,
\end{array}
$$

where $\widehat{S}_{\mp}$ are boundary operators which, by their action on the wave function at the edges of the interval $(-d$ and respectively $+d)$ impose on the solutions of Eq. (1) appropriate boundary conditions. If one assumes symmetric boundary conditions, the solutions of Eq. (1) will also be symmetric or antisymmetric: $\cos z k$ or $\sin z k$, respectively. More generally, if the boundary conditions are asymmetric,

\footnotetext{
${ }^{*}$ e-mail: henpusz@main.amu.edu.pl
} 
we expect the general solution of Eq. (1) to be a superposition of the preceding symmetric and antisymmetric functions. Thus, we write the superposition in the following form:

$$
u_{k}^{-}(z)=\left[\widehat{S}_{-} \sin z k\right]_{-d} \cos z k-\left[\widehat{S}_{-} \cos z k\right]_{-d} \sin z k,
$$

where the expressions in square brackets as coefficients of the superposition have properly postulated forms to ensure that the superposition ( $3 a$ ) fulfils the boundary equation (2a) automatically. Now, we shall be referring to an expression for the wave function that automatically fulfils a well defined boundary condition as a form adjoined to the boundary condition in question; thus, formula (3a) is adjoined to the condition (2a). Similarly, we readily find that the wave function adjoined to the condition ( $2 \mathrm{~b}$ ) has the following form:

$$
u_{k}^{+}(z)=\left[\widehat{S}_{+} \sin z k\right]_{+d} \cos z k-\left[\widehat{S}_{+} \cos z k\right]_{+d} \sin z k .
$$

We note that in either of the two above expressions the coefficients of the superposition (square brackets) are independent of the coordinate $z$ since (after carrying out the indicated operation $\widehat{S}_{\mp}$ on a given function) they are calculated in a well defined point (edge) of the interval. Consequently, we easily find that in both cases

$$
\frac{\mathrm{d}^{2}}{\mathrm{~d} z^{2}} u_{k}^{ \pm}(z)=-k^{2} u \frac{ \pm}{k}(z)
$$

meaning that each of the postulated superpositions (3) fulfils the initial Schrödinger equation with the corresponding energy

$$
E(k)=V+k^{2} \text {. }
$$

One still needs to know the values permitted for the wave number $k$. They are determined by the characteristic equalion which is obtained by inserting one of the solutions postulated (no matter which) into that boundary condition which is non-adjoined thereto (e.g. (3a) into (2b), or (3b) into (2a)). In both cases we get the same equality

$$
\left[\widehat{S}_{+} \cos z k\right]_{+d}\left[\widehat{S}_{-} \sin z k\right]_{-d}-\left[\widehat{S}_{+} \sin z k\right]_{+d}\left[\widehat{S}_{-} \cos z k\right]_{-d}=0 \text {. }
$$

This equation gives the spectrum of permitted values of $k$ we were searching for. We thus have closed the procedure of solving our initial problem.

\section{Application: Sturm-Liouville boundary conditions}

Now we shall illustrate how this method works by considering a simple example. Let the boundary conditions have the following Sturm-Liouville form:

$$
\begin{array}{ll}
\widehat{S}_{-} u(z) \equiv\left(\frac{\mathrm{d}}{\mathrm{d} z}-A\right) u(z)=0 & \text { for } \quad z=-d, \\
\widehat{S}_{+} u(z) \equiv\left(\frac{\mathrm{d}}{\mathrm{d} z}+B\right) u(z)=0 & \text { for } z=+d,
\end{array}
$$

leading to the following expressions: 


$$
\begin{aligned}
& {\left[\widehat{S}_{-} \sin z k\right]_{-d}=k \cos d k+A \sin d k} \\
& {\left[\widehat{S}_{-} \cos z k\right]_{-d}=k \sin d k-A \cos d k} \\
& {\left[\widehat{S}_{+} \sin z k\right]_{+d}=k \cos d k+B \sin d k} \\
& {\left[\hat{S}_{+} \cos z k\right]_{+d}=-(k \sin d k-B \cos d k) .}
\end{aligned}
$$

Consequently, Eq. (3a) takes the form

$$
\begin{aligned}
& u_{k}^{-}(z)=(k \cos d k+A \sin d k) \cos z k-(k \sin d k-A \cos d k) \sin z k \\
& \equiv k \cos (z+d) k+A \sin (z+d) k .
\end{aligned}
$$

and the solution ( $3 \mathrm{~b})$ becomes

$$
\begin{aligned}
u_{k}^{+}(z) & =(k \cos d k+B \sin d k) \cos z k+(k \sin d k-B \cos d k) \sin z k \\
& \equiv k \cos (z-d) k-B \sin (z-d) k .
\end{aligned}
$$

These are equivalent forms representing solutions of the Schrödinger equation (1); of course, both above solutions are determined with accuracy to their normalization and dimensionality constants. Formally, the difference between the solutions resides in the fact that the solution (8) was explicitly expressed in terms of the boundary parameter $A$, whereas the solution (9) is expressed by $B$. Obviously, this difference results from the circumstance that these solutions had already been adjoined, respectively, with the boundary equation (7a) or (7b). We easily find that Eq. (6) takes the form

$$
\begin{aligned}
& (k \sin d k-B \cos d k)(k \cos d k+A \sin d k) \\
& \quad+(k \cos d k+B \sin d k)(k \sin d k-A \cos d k)=0 .
\end{aligned}
$$

Let us now recover the respective classical results one can find in the standard quantum-mechanical textbooks. With this aim let us introduce the scattering phases [1] by postulating the following equalities:

$$
\begin{aligned}
& k \equiv c_{-} \sin \delta_{-}, \\
& A \equiv c_{-} \cos \delta_{-},
\end{aligned}
$$

for the boundary condition at $z=-d$, and similarly

$$
\begin{aligned}
& k \equiv c_{+} \sin \delta_{+}, \\
& B \equiv c_{+} \cos \delta_{+},
\end{aligned}
$$

for the respective boundary condition at $z=+d$. From these equations one easily finds

$$
\tan \delta_{-}=\frac{k}{A}, \quad c_{-}^{2}=k^{2}+A^{2}
$$

and

$$
\tan \delta_{+}=\frac{k}{B}, \quad c_{+}^{2}=k^{2}+B^{2}
$$


Now, if Eqs. (11) are inserted into Eq. (8), and respectively - Eqs. (12) into Eq. (9), the solutions of the Schrödinger equation take the following forms:

$$
\begin{aligned}
& u_{k}^{-}(z)=c_{-} \sin \left[(z+d) k+\delta_{-}\right] \quad \text { and } \\
& u_{k}^{+}(z)=-c_{+} \sin \left[(z-d) k-\delta_{+}\right]
\end{aligned}
$$

while the characteristic equation (10) now reads

$$
\sin \left(2 d k+\delta_{+}+\delta_{-}\right)=0 .
$$

These are standard classical results known from the textbooks (see e.g. $[2,3]$ ).

This example visualizes the beauty and simplicity of our method of finding the solutions of the Schrölinger equation by representing them in the form of functions adjoined to the boundary conditions. Moreover, let us note that the advantage of our approach resides in the fact that (by Eqs. (13)) we were able to relate straightforwardly the scattering phases $\left(\delta_{\mp}\right)$ with their respective boundary parameters $(A / B)$. Another advantage of the method is that it allows (by employing simultaneously both our solutions $u_{k}^{-}(z)$ and $\left.u_{k}^{+}(z)\right)$ to calculate directly the respective Green function (see [2]).

\section{Case of real even potential}

The above proposed method of boundary-adjoined functions is sufficiently general to be applied in other boundary-value problems provided their Hamiltonian possesses the property of symmetricity with respect to the inversion of the coordinate $z$. Thus, let

$$
\left[-\frac{\mathrm{d}^{2}}{\mathrm{~d} z^{2}}+V(z)\right] u(z)=E u(z)
$$

and let the boundary conditions a.gain be given by Eqs. (2a) and (2b). Let the particular solutions of (16) be given by certain symmetric functions $u_{\mathrm{s}}(z)$ and antisymmetric functions $u_{\mathrm{a}}(x)$; their form is obviously "dictated" by the nature of the potential $V(z)$ endowed with the property of invariance with respect to inversion of the coordinate $z$ :

$$
V(z)=V(-z) \text {. }
$$

The general solution of (16) can then be represented in the form of a superposition of these particular solutions

$$
u_{k}(z)=\left[\widehat{S}_{-} u_{\mathrm{a}}(z)\right]_{-d} u_{\mathrm{s}}(z)-\left[\widehat{S}_{-} u_{\mathrm{s}}(z)\right]_{-d} u_{\mathrm{a}}(z) .
$$

Obviously, expression (18) automatically fulfils the boundary condition (2a), whereas the other boundary condition (2b) leads to the following characteristic equation:

$$
\left[\widehat{S}_{+} u_{\mathrm{s}}(z)\right]_{+d}\left[\widehat{S}_{-} u_{\mathrm{a}}(z)\right]_{-d}-\left[\widehat{S}_{+} u_{\mathrm{a}}(z)\right]_{+d}\left[\widehat{S}_{-} u_{\mathrm{s}}(z)\right]_{-d}=0 .
$$

The energy corresponding to the solution (18) is to be obtained simply by insertion of (18) into (16). 


\section{Outlooks}

The method proposed provicles explicit forms of boundary-adjoined wave functions and leads straightforwardly to a characteristic equation resulting from the boundary conditions. This is especially noticeable for asymmetric boundary conditions when we gain a specific simplification of the procedure. We expect that this method may turn out to be beneficial with regard to the recently growing interest in one-dimensional quantum systems. For example, it may facilitate the calculations of the respective density of slates functions or effects due to an electron tunnelling through imsulating barriers [4]. Although, in general, it is possible to solve respective problems numerically, analytic calculations still provide insight valuable in understanding the physical mechanisms responsible for quantum effects. It is also evident that the proposed method of using the boundary-adjoined functions can be applied to the study of other (besides Schrödinger's) differential equations. These matters will be dealt with in a separate paper.

\section{Acknowledgments}

The present work was supported by grant No. 2 P03B 07916 from the Committee for Scientific Research.

\section{References}

[1] I. Białynicki-Birula, M. Cieplak, J. Kaminiski, Theory of Quanta, Oxford University Press, New York 1992.

[2] L.I. Schiff, Quantum Mechanics, 3rd ed, McGraw-Hill, New York 1968.

[3] L. Landau, E.M. Jifshitz, Quantum Mechanics. Nonrelativistic Theory, PWN, Warsaw 1979.

[4] C.B. Duke, Tunnelling in Solids, Solid State Physics, Suppl., Vol. 10, Acautemic Press, New York 1969. 